\title{
Design of Potential Antimalarial Agents Based on a Homology Model of Plasmodium falciparum Glucose-6-Phosphate Dehydrogenase ${ }^{+}$
}

\author{
Caterina Pont ${ }^{1}$, Nelson Alencar ${ }^{2,3}$, Irene Sola ${ }^{1}$, María Linares 4, Luca Di Palma 1, \\ Carla Barbaraci ${ }^{1}$, Cristina Sampedro ${ }^{1}$, Jordi Juárez-Jiménez ${ }^{2}$, Paloma Abad ${ }^{4,5}$, \\ Susana Pérez-Benavente ${ }^{5}$, Jerónimo Lameira ${ }^{3}$, José M. Bautista ${ }^{4,5}$, F. Javier Luque ${ }^{2}$ and \\ Diego Muñoz-Torrero ${ }^{1, *}$
}

1 Laboratory of Pharmaceutical Chemistry (CSIC Associated Unit), Faculty of Pharmacy and Food Sciences and Institute of Biomedicine (IBUB), University of Barcelona (UB), Av. Joan XXIII 27-31, E-08028 Barcelona, Spain; aitak1989@gmail.com (C.P.); irenesola872@gmail.com (I.S.); lucadipalma92@gmail.com (L.D.P.); carlabarbaraci@rocketmail.com (C.B.); cristinasampedrogarcia@gmail.com (C.S.);

2 Department of Nutrition, Food Science and Gastronomy, Faculty of Pharmacy and Food Sciences, and IBUB, University of Barcelona, Av. Prat de la Riba 171, E-08921 Santa Coloma de Gramenet, Spain; nelsonalencar@gmail.com (N.A.); jordi.juarez@gmail.com (J.J.-J.); fjluque@ub.edu (F.J.L.)

3 Laboratório de Planejamento e Desenvolvimento de Fármacos-LPDF, Instituto de Ciências Exatas e Naturais-ICEN, Universidade Federal do Pará-UFPA, Av. Augusto Correa, № 1-Bairro: Guamá, Cep: 66.075-900 Belém-Pará, Brazil; lameira@ufpa.br

4 Research Institute Hospital 12 de Octubre, Avda. de Cordoba s/n, 28041 Madrid, Spain; marialinares@vet.ucm.es (M.L.); paloma.abadg@gmail.com (P.A.); jmbau@ucm.es (J.M.B.)

5 Department of Biochemistry and Molecular Biology, Universidad Complutense de Madrid, Facultad de Veterinaria, Ciudad Universitaria, 28040-Madrid, Spain; susipz@ucm.es

* Correspondence: dmunoztorrero@ub.edu

+ Presented at the 1st Molecules Medicinal Chemistry Symposium, Barcelona, Spain, 8 September 2017.

Published: 18 October 2017

There currently exists a dire need for safe and inexpensive new antimalarial drugs, which are effective against multiple life cycle stages of Plasmodium falciparum, and act through mechanisms that differ from those of the available drugs to prevent drug resistance. The enzyme glucose-6-phosphate dehydrogenase (G6PD) of $P$. falciparum has emerged as a promising target for antimalarial drug discovery, due to its key role in parasite development and survival and its protective effect against malaria infection observed under human G6PD deficiency conditions. Here, we describe the construction of a homology model of PfG6PD, which has enabled the identification of key structural differences as compared with the human enzyme. We have exploited these changes to rationally design a novel family of substrate analog-based inhibitors that can be endowed with selectivity towards PfG6PD. Several compounds display micromolar affinity, good selectivity, and low cytotoxicity, but weak antiplasmodial activity in phenotypic assays, likely as a result of a poor internalization of the compounds in the parasite cell. Future hit optimization should focus on improving the physicochemical/pharmacokinetic properties of this class of compounds.

Acknowledgments: This work was supported by Ministerio de Economía y Competitividad (MINECO) (SAF2014-57094-R, BIO2013-44565R and BIO2016-77430R) and Generalitat de Catalunya (GC) (2014SGR52, 2014SGR1189). Fellowships from GC to C.P and I.S.; from FIS to J.J.-J.; from Lifelong Learning Programme/Erasmus to L.D.P. and C.B.; and from Comunidad de Madrid (PEJ16/MED/AI-0792) to P.A. are acknowledged. Thanks are also due to the ICREA Academia program (to F.J.L.), and to the Unión Iberoamericana de Universidades (to D.M.-T., F.J.L. and J.M.B.). The Center for Scientific and Academic Services of Catalonia (CESCA) is acknowledged for providing access to computational facilities. M.L. held a postdoctoral Fellowship of the Spanish Ministry of Economy and Competitiveness (FPDI-2013-16409). 
Author Contributions: C.P., I.S., L.D.P., C.B. and C.S. performed the synthetic work; N.A. and J.J.-J. performed the computational work; and M.L., P.A. and S.P.-B. performed the biological work. D.M.-T supervised the synthetic work; F.J.L. and J.L. supervised the computational work; and J.M.B. supervised the biological work. F.J.L. and D.M.-T. conceived the project.

Conflicts of Interest: The authors declare no conflicts of interest

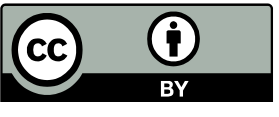

(C) 2017 by the authors. Licensee MDPI, Basel, Switzerland. This article is an open access article distributed under the terms and conditions of the Creative Commons Attribution (CC BY) license (http://creativecommons.org/licenses/by/4.0/). 